\title{
Coaching und Supervision im schulischen Kontext
}

\section{A. Schreyögg}

Online publiziert: 6. Mai 2015

(C) Springer Fachmedien Wiesbaden 2015

Wir haben schon im Jahr 1995 ein Heft von OSC herausgegeben mit dem Titel „Supervision und Organisationsentwicklung in der Schule“ (2. Jg. 2/1995), obwohl sich diese beiden Formate noch lange Zeit als Fremdkörper in schulischen Kontexten erwiesen. Als „,clinical supervision“ konnte sich die Supervision seit Beginn des Zwanzigsten Jahrhunderts nur in der Sozialarbeit und später in der Psychotherapie als selbstverständliches Instrument der Qualitätskontrolle verankern. In schulischen Zusammenhängen tauchte sie vergleichsweise spät in Erscheinung. Die ersten Versuche in den 1950er Jahren akzentuierten primär Defizite von Lehrkräften auf psychoanalytischer Basis, sodass sich nachfolgend geradezu Aversionen gegen die Supervision gebildet haben. In schulischen Milieus wurde sie weithin als therapieähnliches Verfahren begriffen, das man für sich zumeist als unpassend bzw. kränkend ablehnte. Erst zu Beginn des 21. Jahrhunderts reifte in manchen deutschen Kultusbehörden die Einsicht, man könne durch Supervision als Maßnahme der Personalentwicklung die Lehrerschaft in ihren Potenzialen fördern und vielleicht sogar Innovationen im schulischen Gesamt in Bewegung bringen.

Zu diesem Thema haben wir bereits im Jahr 2000 ein Heft (7. Jg. 3/2000) gemacht. Vor allem die vielen Stress- und Burnout-Phänomene in Lehrerkollegien zogen eine erhöhte Bereitschaft für die Supervision und damit auch für die Ausbildung von Supervisoren nach sich. Im Zuge dieser Entwicklung tauchten auch erste Versuche mit Coaching auf. Es sprach sich nämlich herum, dass auch Schulleiter und sogar Schulaufsichtsbeamte von beruflicher Beratung profitieren könnten. Dies ergab sich insbesondere dann, wenn sie mit Konflikten in schulischen Organisationen konfrontiert waren (Schreyögg 2000, 2008; Schreyögg und Lehmeier 2003). Im vorliegenden Heft geben wir nun Kostproben von der weiteren Entwicklung, die „Coaching

Dr. A. Schreyögg $(\bowtie)$

Breisgauer Str. 29,

14129 Berlin, Deutschland

E-Mail: info@schreyoegg.de 
und Supervision im schulischen Kontext" aufweisen. Die beiden Formate haben sich nämlich jetzt besser verankert, sodass die behandelten Fragestellungen zunehmend facettenreicher und spezialisierter als früher wurden.

Im ersten Beitrag berichten Andrea Blendinger und Susanne Tober, Schulpsychologinnen in Bayern, wie Supervision als „Instrument zur Stärkung von Resilienz“ in der „Gruppensupervision von Lehrkräften“ genutzt werden kann. Sie fächern zunächst einige Resilienzfaktoren auf, sodann zeigen sie an Praxisbeispielen, wie Lehrkräfte gestärkt werden können. Abschließend empfehlen sie, Gruppensupervision als obligatorisches Mittel an Schulen zu verankern. Der nachfolgende Aufsatz von Irene Terzer Prader, Supervisorin und Coach in Südtirol, thematisiert die Position von Schulleiterstellvertreter/innen. Die Autorin beschreibt, dass die Positionsinhaber laufend zwischen der Rolle als Sprachrohr für das Direktorat und als Prellbock für das Kollegium fungieren müssen. Dadurch befänden sie sich in einem permanenten Balance-Zustand, der sie gelegentlich zu zerreiben droht. Schule als gesellschaftliche Institution stellt für alle Menschen einen höchst relevanten Lebensraum dar. In diesem Verständnis präsentieren Markus Prummer und Anna Hasmüller ein „Mobbing-Präventions-Projekt“. Sie plädieren dafür, dass auf verschiedenen Ebenen des Schulsystems Entwicklungsprozesse für einen geplanten organisatorischen Wandel in Gang gesetzt werden. Dieser ziele dann nicht einfach nur auf Verhaltensänderungen einzelner, sondern auf einen Wandel der gesamten Schulkultur.

Der nachfolgende Beitrag von Ulrike Steiner entstammt zwar nicht dem schulischen Milieu, er behandelt aber ein Thema, das für Supervision und Coaching in jedweder Organisation relevant ist, nämlich die Loyalität des organisationsinternen Beraters zu der Organisation, der er selbst angehört. Die Autorin, Mitarbeiterin in einer großen Bundesbehörde, rollt die Dreiecks-Beziehung zwischen Coach, Coaching-Klient und Organisation sehr facettenreich auf. Sie benennt vor allem Konfliktpotenziale und gibt Anregungen zum Umgang mit ihnen.

Der erste Praxisbericht widmet sich wieder schulischen Belangen. Hier beschreiben Susanne Haas-Breidung und Sabine Kurrer „interkulturelle Supervision am Beispiel einer Elternlotsengruppe“. Die beiden Autorinnen präsentieren ein Modellprojekt in Nürnberg, an dem Eltern aus fünf Nationen teilgenommen haben. Sie zeigen, wie die Supervision durch die kulturelle Dimension einerseits, aber auch durch individuelle Belange andererseits eingefärbt wurde. Sie zeigen aber auch die Grenzen solcher Beratung auf. Beim nächsten Praxisbericht bleiben wir im interkulturellen Rahmen. Hier geht es um „Spiegelphänomene in der interkulturellen Teamsupervision". Katharina Behrens beschreibt, wie sich noch nicht bewusste oder noch nicht verbalisierte Spannungen und Ressourcen in der Supervision als „Kommunikationsgefäß“" manifestieren können und wie sie dadurch der Bearbeitung zugänglich werden. Der dritte Praxisbericht von Christine Kentzler widmet sich einem Thema negativer Führungshaltung. Als ,abusive supervision“, d. h. missbräuchliche Führung, beschreibt sie ein Phänomen, das keineswegs nur als singuläres Ereignis zu werten ist, sondern von dem prinzipiell ein gesamtes organisatorisches System im Sinne chronifizierter Kränkungen infiltriert ist. Neben den Ursachen zeigt die Autorin auch Möglichkeiten der Gegenwehr auf.

Im Diskurs präsentiere ich, Astrid Schreyögg, auch ein Thema mit systemischem Charakter. Dabei geht es um „Fehlerkultur im Kinderschutz“. In einem Vortrag für 
die „Bundesinitiative frühe Hilfen“ hatte ich darauf aufmerksam gemacht, dass Kinder nicht nur wegen einzelner Personen zu Schaden kommen, sondern dass das gesamte institutionalisierte Kinderschutz-System einer Neubesinnung und Reorganisation bedarf.

\section{Literatur}

Schreyögg, A. (Hrsg.). (2000). Supervision und Coaching für die Schulentwicklung. Bonn: DPV. Schreyögg, A. (Hrsg.). (2008). Konfliktcoaching und Konfliktmanagement in Schulen. Bonn: DPV. Schreyögg, A., \& Lehmeier, H. (Hrsg.). (2003). Personalentwicklung in der Schule. Bonn: DPV. 